\title{
Precision public health through clinic-based syndromic surveillance in communities
}

\section{Ta-Chien Chan, Yung-Chu Teng, Yen-Hua Chu, Tzu-Yu Lin}

\author{
Academia Sinica, Taipei City, Taiwan
}

Objective

Sentinel physician surveillance in the communities has played an important role in detecting early aberrations in epidemics. The traditional approach is to ask primary care physicians to actively report some diseases such as influenza-like illness (ILI), and hand, foot, and mouth disease (HFMD) to health authorities on a weekly basis. However, this is labor-intensive and time-consuming work. In this study, we try to set up an automatic sentinel surveillance system to detect 23 syndromic groups in the communites.

\section{Introduction}

In December 2009, Taiwan's CDC stopped its sentinel physician surveillance system. Currently, infectious disease surveillance systems in Taiwan rely on not only the national notifiable disease surveillance system but also real-time outbreak and disease surveillance (RODS) from emergency rooms, and the outpatient and hospitalization surveillance system from National Health Insurance data. However, the timeliness of data exchange and the number of monitored syndromic groups are limited. The spatial resolution of monitoring units is also too coarse, at the city level. Those systems can capture the epidemic situation at the nationwide level, but have difficulty reflecting the real epidemic situation in communities in a timely manner. Based on past epidemic experience, daily and small area surveillance can detect early aberrations. In addition, emerging infectious diseases do not have typical symptoms at the early stage of an epidemic. Traditional disease-based reporting systems cannot capture this kind of signal. Therefore, we have set up a clinic-based surveillance system to monitor 23 kinds of syndromic groups. Through longitudinal surveillance and sensitive statistical models, the system can automatically remind medical practitioners of the epidemic situation of different syndromic groups, and will help them remain vigilant to susceptible patients. Local health departments can take action based on aberrations to prevent an epidemic from getting worse and to reduce the severity of the infected cases.

\section{Methods}

We collected data on 23 syndromic groups from participating clinics in Taipei City (in northern Taiwan) and Kaohsiung City (in southern Taiwan). The definitions of 21 of those syndromic groups with ICD-10 diagnoses were adopted from the International Society for Disease Surveillance (https://www.surveillancerepository.org/icd-10-cm-master-mapping-reference-table). The definitions of the other two syndromic groups, including dengue-like illness and enterovirus-like illness, were suggested by infectious disease and emergency medicine specialists. An enhanced sentinel surveillance system named "Sentinel plus" was designed for sentinel clinics and community hospitals. The system was designed with an interactive interface and statistical models for aberration detection. The data will be computed for different combinations of syndromic groups, age groups and gender groups. Every day, each participating clinic will automatically upload the data to the provider of the health information system (HIS) and then the data will be transferred to the research team. This study was approved by the committee of the Institutional Review Board (IRB) at Academia Sinica (AS-IRB02-106262, and AS-IRB02-107139). The databases we used were all stripped of identifying information and thus informed consent of participants was not required.

\section{Results}

This system started to recruit the clinics in May 2018. As of August 2018, there are 89 clinics in Kaohsiung City and 33 clinics and seven community hospitals in Taipei City participating in Sentinel plus. The recruiting process is still ongoing. On average, the monitored volumes of outpatient visits in Kaohsiung City and Taipei City are 5,000 and 14,000 per day. Each clinic is provided one list informing them of the relative importance of syndromic groups, the age distribution of each syndromic group and a timeseries chart of outpatient rates at their own clinic. In addition, they can also view the village-level risk map, with different alert colors. In this way, medical practitioners can know what's going on, not only in their own clinics and communities but also in the surrounding communities. The Department of Health (Figure 1) can know the current increasing and decreasing trends of 23 syndromic groups by red and blue color, respectively. The spatial resolution has four levels including city, township, village and clinic. The map and bar chart represent the difference in outpatient rate between yesterday and the average for the past week. The line chart represents the daily outpatient rates for one selected syndromic group in the past seven days. The age distribution of each syndromic group and age-specific outpatient rates in different syndromic groups can be examined.

SDS Annual Conference Proceedings 2019. This is an Open Access article distributed under the terms of the Creative Commons AttributionNoncommercial 4.0 Unported License (http://creativecommons.org/licenses/by-nc/3.0/), permitting all non-commercial use, distribution, and reproduction in any medium, provided the original work is properly cited. 


\section{Conclusions}

Sentinel plus is still at the early stage of development. The timeliness and the accuracy of the system will be evaluated by comparing with some syndromic groups in emergency rooms and the national notifiable disease surveillance system. The system is designed to assist with surveillance of not only infectious diseases but also some chronic diseases such as asthma. Integrating with external environmental data, Sentinel plus can alert public health workers to implement better intervention for the right population.

\section{Acknowledgement}

This research was supported by a grant titled "Multidisciplinary Health Cloud Research Program: Technology Development and Application of Big Health Data" from Academia Sinica and a grant titled "Implementing Integrated Surveillance Network of Dengue Fever" from the National Health Research Institute, Taiwan and a grant from the Department of Health, Taipei City government. We would like to thank the Departments of Health in Kaohsiung and Taipei, as well as Taipei Medical Association for help in recruiting the participating clinics and hospitals.

\section{References}

1. James W. Buehler AS, Marc Paladini, Paula Soper, Farzad Mostashari: Syndromic Surveillance Practice in the United States: Findings from a Survey of State, Territorial, and Selected Local Health Departments. Advances in Disease Surveillance 2008, 6(3).

2. Ding Y, Fei Y, Xu B, Yang J, Yan W, et al. 2015. Measuring costs of data collection at village clinics by village doctors for a syndromic surveillance system — a cross sectional survey from China. BMC Health Serv Res. 15, 287. PubMed https://doi.org/10.1186/s12913-015-0965-2

3. Kao JH, Chen CD, Tiger Li ZR, Chan TC, Tung TH, et al. 2016. The Critical Role of Early Dengue Surveillance and Limitations of Clinical Reporting -- Implications for Non-Endemic Countries. PLoS One. 11(8), e0160230. PubMed https://doi.org/10.1371/journal.pone.0160230

4. Chan TC, Hu TH, Hwang JS. 2015. Daily forecast of dengue fever incidents for urban villages in a city. Int J Health Geogr. 14, 9. PubMed https://doi.org/10.1186/1476-072X-14-9

5. Chan TC, Teng YC, Hwang JS. 2015. Detection of influenza-like illness aberrations by directly monitoring Pearson residuals of fitted negative binomial regression models. BMC Public Health. 15, 168. PubMed https://doi.org/10.1186/s12889-015$\underline{1500-4}$

6. Ma HT. Syndromic surveillance system for detecting enterovirus outbreaks evaluation and applications in public health. Taipei, Taiwan: National Taiwan University; 2007.

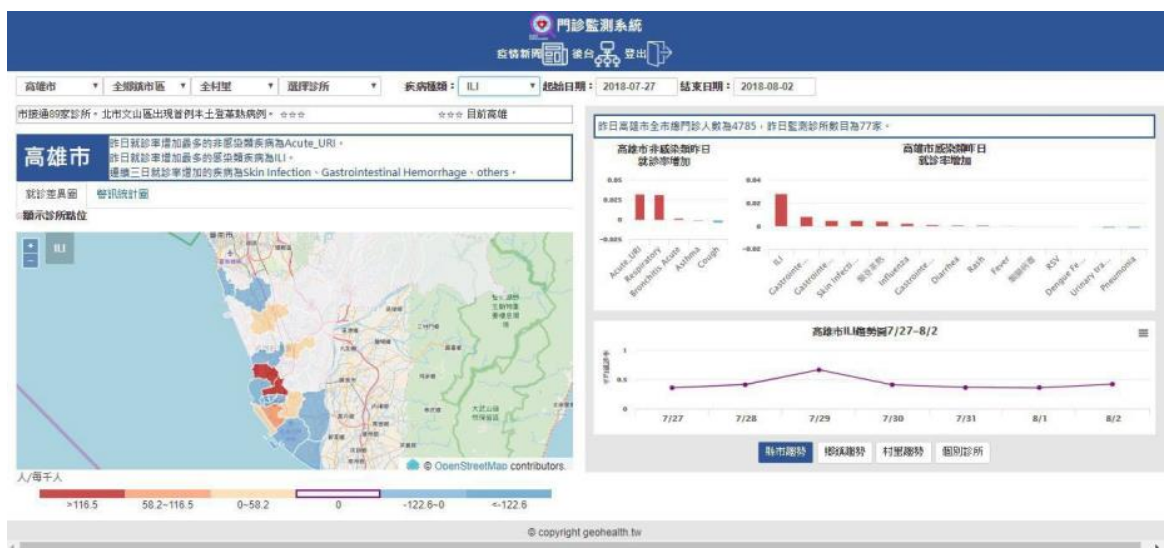

Figure 1. The Department of Health dashboard in Sentinel plus

SDS Annual Conference Proceedings 2019. This is an Open Access article distributed under the terms of the Creative Commons AttributionNoncommercial 4.0 Unported License (http://creativecommons.org/licenses/by-nc/3.0/), permitting all non-commercial use, distribution, and reproduction in any medium, provided the original work is properly cited. 